Arthroskopie 2011 $\cdot 24: 182-182$

DOI 10.1007/s00142-010-0624-x

Online publiziert: 3. Juli 2011

(c) Springer-Verlag 2011

\author{
A.B. Imhoff ${ }^{1} \cdot$ S. Rupp ${ }^{2}$ \\ ${ }^{1}$ Abteilung und Klinik für Sportorthopädie, Klinikum rechts der Isar, \\ Technische Universität München \\ ${ }^{2}$ Fachklinik für Konservative Orthopädie Blieskastel
}

\title{
Studienplanung und Praxisorganisation
}

Sehr geehrte Leserinnen und Leser,

jeder Student und jeder junge Forscher kommt irgendwann dazu, ein Forschungskonzept oder ein Forschungsprojekt zu schreiben. Unnötige Angst und Unsicherheit können durch strukturierte Informationshilfen und Checklisten auf einfache Weise vermieden werden.

\section{- Bereits der Prozess des Konzeptschreibens ist der erste Schritt zum erfolgreichen Projekt.}

Allerdings muss das Ziel immer wieder hinterfragt werden (Eintagsfliege oder längerfristiges Forschungsvorhaben/ Habilitation). Mit einem guten Leitfaden, Sorgfalt, Struktur des Arbeitens und der Akzeptanz einer Lernkurve und von Fehlern kann ein erstes Konzept leicht erreicht werden. Mentoren auf der einen, journalistische Werkzeuge, Tipps und Tricks auf der anderen Seite sind die Hilfsmittel zum Gelingen. Zu den Werkzeugen gehören strukturierte Richtlinien, wie sie von der NIH, DFG oder auch der AGA vorgegeben werden und die einer späteren Begutachtung entgegenkommen.

Das AGA-Research-Komitee hatte am letzten AGA-Kongress 2010 im Rahmen eines Instruktionskurses die wichtigsten Aspekte bei der Erarbeitung und Durchführung eines Forschungsprojekts vorgestellt und seither mit vielen jungen Forschern diskutiert. Die wichtigsten Beiträge haben wir in diesem Heft zusammengestellt.

Der erste Artikel stammt von Christian Lattermann, der aus amerikanischer Sicht (University of Kentucky) einen Leit- faden mit den wesentlichen Elementen der Planung und Durchführung von Forschungsprojekten beschreibt und anhand von Flussdiagrammen illustriert. Ergänzt wird diese Thematik durch Thore Zantop, früher Universität Münster, jetzt Sporthopaedicum Straubing, der die Probleme bei der Publikation von Forschungsergebnissen aufnimmt. Peter Brucker von der TU München versucht in seinem Beitrag, die beiden Begriffe Evidence- bzw. Eminence-based Medicine auseinander zu halten: Während die Evidence-based Medicine wissenschafltiche Beweise und Nachweise fordert, beruht die Eminence-based Medicine auf Meinungen und Erfahrungen von Experten, die nicht zwingend den Kriterien der Evidence-based Medicine entsprechen müssen. Ein sehr aktuelles Thema des Urheberrechts und eng verknüpft des Plagiats wird von der Berliner Autorengruppe Sebastian Kopf und Roland Becker zusammen mit Carsten Heimann vom Lehrstuhl für Bürgerliches Recht in Magdeburg differenziert aufgearbeitet.

Der zweite Teil des aktuellen Hefts widmet sich in 2 Beiträgen der Praxisorganisation. Am Beispiel eines Operationszentrums mit Ambulanz/Praxis, Operationszentrum für ambulante und stationäre Operationen und Anbindung an eine Privat- und Belegklinik werden die Möglichkeiten der Optimierung der Abläufe und Prozesse im Ambulanz-/ Praxis- und Operationsbetrieb dargestellt (G. Wasmer). Der zweite Beitrag betrifft die arthroskopische Behandlung als stationäre Leistung im DRG-System ( $M i$ chael M. Kaufmann).
Mit diesem Heft wollen wir den Lesern und AGA-Mitgliedern einen kurzen Leitfaden anbieten, der die wesentlichen Hürden auf dem Weg zu einem erfolgreichen Forschungsprojekt anspricht und ein paar Tipps gibt, diese Hürden zu umgehen.

Ihre

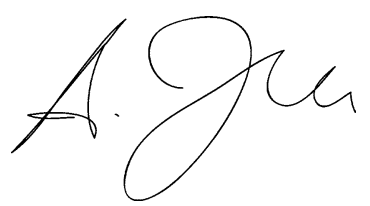

Andreas Imhoff und

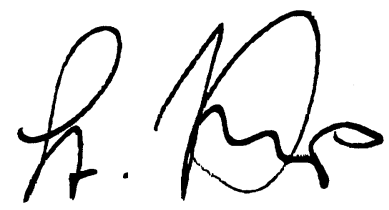

Stefan Rupp

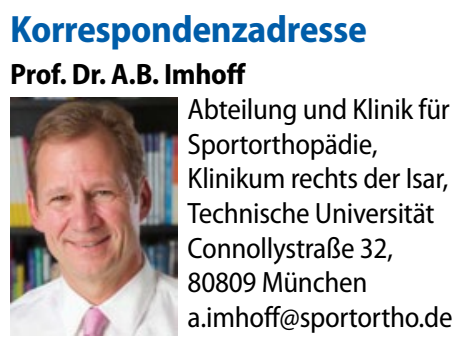

Interessenkonflikt. Der korrespondierende Autor gibt an, dass kein Interessenkonflikt besteht.

\section{Literatur}

1. DFG (Deutsche Forschungsgemeinschaft) Mein erster Antrag - Nützliche Hinweise rund um die Antragstellung. http://www.dfg.de/foerderung/ grundlagen_dfg_foerderung/wissenschaftliche karriere/erstantrag/index.html 